\section{G450(P) HYPERIMMUNOGLOBULINE E SYNDROME CASE PRESENTATION}

H El-Salheen, A Badi, I Matoug. Paediatrics ID and Immunology, Benghazi Children Hospital, Benghazi, Libya

\subsection{6/archdischild-2015-308599.404}

Background Hyperimmunoglobuline E syndrome (HIES), also known as Job Syndrome, is a rare primary immuneodeficiency disorder characterised by recurrent eczema, skin abscesses, lung infections, eosinophilia and high serum level of IgE. Two forms of HIES have been described, type 1(Autosomal Dominant) and type 2 (Autosomal Recessive) which is more severe. These two forms share overlapping clinical and laboratory features. However, they also exhibit distinct clinical manifestations, courses and outcomes. This paper will present our experience with this rare disease in our new Immunology unit.

Methods Six cases were diagnosed between end 2008 to early 2014, depending on clinical features, laboratory and radiological evaluation.

Results Two cases of HIES presented before their second birthday and the other four before their fifth. Two cases were brothers. Male to female ratio was 2:1.

Each case initially presented with different chronic infections. All have coarse features, eczema, high IgE (>2500 iu/ $\mathrm{ml}$ ), high eosinophil count and recurrent oral thrush. Five of the six cases have onychomycosis. Three cases had extensive varicella eruption. Infections were treated according to the international guidelines. Chest $\mathrm{x}$-ray showed lung cysts in two cases, three cases had no lung changes and one patient refused $\mathrm{x}$-ray and any follow up. Echocardiogram was normal in five cases. Serum Immunoglobulins, $\mathrm{T}$ and $\mathrm{B}$ lymphocyte subset were normal for four cases. The other two cases, one of them showed low T helper cell and low CD4 percentage with normal total count, and the other case showed low natural killer cell CD56 percentage and high count CD19, CD4, CD8, CD3 with normal percentage. All cases were put on oral prophylactic Trimethoprim and regular follow up except one who refused (Figure 1).

Conclusion The rising awareness among junior doctors and early referral of cases with symptoms to suggest primary immunodeficiency disorders help to diagnose and treat cases of HIES (Job Syndrome) which used to be missed and arrived to hospital in serious or terminal state. The incidence rate of Job syndrome internationally is rare. Further studies are needed to estimate the incidence rate and prevalence in different areas of our country.

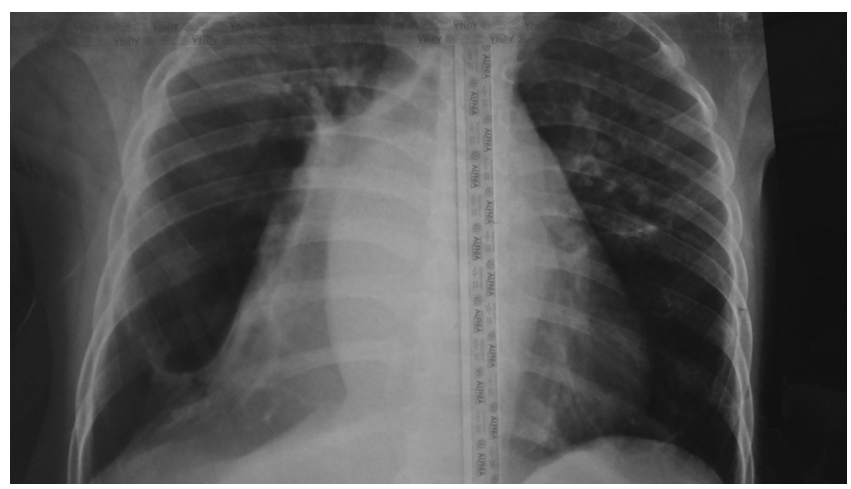

Abstract G450(P) Figure 1

\section{G451(P) IMPROVING SCHOOL ATTENDANCE AT A SPECIAL NEEDS SCHOOL - A SERVICE EVALUATION}

${ }^{1} \mathrm{Z}$ Arslan, ${ }^{2} \mathrm{P}$ Julies. ${ }^{1}$ Neonates, Barnet Hospital, London, UK; ${ }^{2}$ Community Paediatrics, Royal Free Hospital, London, UK

\subsection{6/archdischild-2015-308599.405}

Introduction Pupils with learning disabilities find it more challenging to learn than their peers. Due to their complex needs, they also have a greater rate of school absenteeism (1). Attendance is vital for learning new skills (target attendance $>95 \%$ ) (2). We looked at medical reasons for school absenteeism at a special needs school in Camden.

Aims To identify medical reasons for school absenteeism during winter months, with a view to introducing targeted interventions to improve attendance.

Methods Retrospective data collection of absentees from school register between September 2013 to March 2014 and clinical case notes review.

Results Out of 229 primary and secondary pupils attending, we included the ones with attendance $<85 \%$. 17\% $(\mathrm{n}=38)$ of the pupils had an attendance of $<85 \%$. The main diagnoses were varying severity of learning disabilities $(41 \% \mathrm{n}=14)$, epilepsy $(26 \% \mathrm{n}=9)$, autism $(21 \% \mathrm{n}=7)$ and cerebral palsy $(12 \% \mathrm{n}=$ 4). Adolescents (aged 14-19) formed the bulk of the group affected (35\%). Illness (unknown 47\%, sleep 15\%, seizures 9\%, others $29 \%)$ were the most prevalent reasons $(67 \% n=19)$ followed by social factors $(21 \% \mathrm{n}=6)$ and medical appointments $(12 \% \mathrm{n}=4)$. No major respiratory illnesses or safeguarding concerns were highlighted in any of the cases. All, except 4 children had named consultants. $73 \%$ had medical reviews in date and had their medical needs identified and treated.

Conclusion Illness (type uncertain) was the main reason for absenteeism. Sleep difficulties and poorly controlled seizures were noted frequently. Unclear coding made further evaluation difficult.

Recommendations School attendance should be made an integral part of school medical reviews.

Absenteeism data should be coded clearly to enable identification medical reasons for absenteeism. School should receive notification from local Accident and Emergency departments when a special needs pupil attends. Targeted interventions to improve attendance can then be implemented.

\section{G452(P) NOT JUST A PAIN IN THE NECK? AN UNUSUAL CASE OF AN INFANT PRESENTING WITH A STIFF NECK, DIAGNOSED WITH SEPTIC ARTHRITIS OF THE ATLANTOAXIAL JOINT AND OSTEOMYELITIS OF THE ODONTOID PEG}

S Murray, C Coleman, M Russell-Taylor. Paediatrics, Stoke Mandeville Hospital, Aylesbury, UK

\subsection{6/archdischild-2015-308599.406}

Primary acute osteomyelitis of the first and second vertebrae is extremely rare in the paediatric population, and there is no literature documenting septic arthritis of the atlantoaxial joint secondary to odontoid osteomyelitis. We present the case of a 10-month-old, well child presenting with a stiff neck, with no clinical features suggestive of infection.

Blood tests revealed a mildly elevated white cell count and CRP, with a normal ESR. Notably his alkaline phosphatase was 
markedly elevated. A lateral cervical spine X-ray excluded bony pathology, but revealed pre-cervical soft tissue swelling. MRI showed extensive signal change in the odontoid peg, along with enhancement and fluid distension of the joint capsule - appearances in keeping with a diagnosis of septic arthritis of the atlanto-axial joint and osteomyelitis of the odontoid peg. The patient was commenced on once-daily intravenous antibiotics for six weeks, before switching to oral therapy for a total of three months. He responded well to treatment and recovered a full range of neck movements. Repeat imaging demonstrated resolution of inflammatory changes, and he was discharged from follow-up.

A high index of suspicion is required for the diagnosis of osteomyelitis, as it has variable presentation and initial investigations may be misleading. This case indicates that nuclear imaging should be done as a matter of urgency in a child who has no appreciable active or passive neck movement to rule out bony pathology, and that although a rare cause of stiff neck in children, odontoid peg osteomyelitis should be considered as a differential diagnosis even in a child who does not look septic. This case also illustrates that odontoid peg osteomyelitis may subsequently lead to septic arthritis of the atlantoaxial joint, which has not been previously described in the literature.

\section{G453(P) MANAGEMENT OF KAWASAKI DISEASE IN A DISTRICT GENERAL HOSPITAL. IS THERE AN OPTIUM ASPIRIN DOSE?}

K Murtagh, F Damda. Department of Paediatrics, University Hospital Lewisham, Lewisham and Greenwich NHS Trust, London, UK

10.1136/archdischild-2015-308599.407

Aims Kawasaki disease (KD) is the commonest cause of paediatric acquired heart disease in the UK. Early treatment significantly reduces coronary artery complications. This study aimed to identify cases of $\mathrm{KD}$ presenting to our district general hospital (DGH). We wished to see how well diagnosis and management adhered to local guidelines.

Methods Retrospective review of case notes of patients admitted to our district general and diagnosed with KD between October 2011 and October 2014. Diagnosis and management was compared to local guideline.

Results Eighteen KD patients of variable age (3 months to 9 years) and ethnicity were identified. Two thirds of cases had incomplete KD. All had a history of fever $>5$ days. Diagnostic features included conjunctivitis (16/18); mucosal changes (15/ $18)$; rash (11/18); cervical lymphadenopathy and peripheral extremity changes (8/18). Irritability was a common feature. BCG scar changes were also present (2/18).

All cases received intravenous immunoglobulin (IVIG) at $2 \mathrm{~g} /$ $\mathrm{kg}$ and high dose aspirin at $30-100 \mathrm{mg} / \mathrm{kg} /$ day except one who received aspirin alone as afebrile at presentation.

Five patients had suspicion of coronary artery dilatation/ prominence on initial echocardiogram. Three subsequently developed coronary artery dilatation all of whom had received IVIG during first week of fever.

Three cases were transferred to tertiary centre for secondline management encompassing repeat IVIG (3/18), infliximab $(2 / 18)$ and corticosteroids (1/18).
Conclusion Two thirds of KD cases were incomplete. Ninetyfour percent had IVIG. Inflammatory markers, laboratory criteria and BCG scar change did not correspond with cardiovascular complications. Dose of aspirin given varied between $30-100 \mathrm{mg} / \mathrm{kg} /$ day depending on tertiary centre recommendation. All patients were managed appropriately. Those that failed to respond were referred to a tertiary centre. This study demonstrates most KD can be managed locally with adherence to clear guidelines and receiving tertiary advice when required. However clearer consensus on high-dose aspirin is needed.

\section{G454(P) RETROSPECTIVE SURVEY OF CHILD PROTECTION CASE CHARACTERISTICS IN AN ACUTE AND COMMUNITY SETTING}

R Sharma, P Mistry. Community Paediatric Department, University Hospitals of Leicester, Leicester, UK

\subsection{6/archdischild-2015-308599.408}

Aim The first principle of safeguarding services stated in the Munro report $2010^{1}$ places the quality of experience and views of the child at the centre. Lord Laming's review in $2009^{2}$ recommended minimum requirements to be met during the safeguarding process. Safeguarding assessments for children occur in both an outpatient (OP) clinic setting and in an acute out of hours $(\mathrm{OOH})$ setting. The aim of this survey was to compare the number, type and outcome of safeguarding assessments between these two settings.

Method Retrospective case-notes review of all cases where a safeguarding examination was completed due to Section 47 concerns from January to March 2013. Those that were referred due to accidental incidents or where there was found to be no safeguarding concern were excluded. Data was obtained on documentation of the process and timeline for assessment as well as the types of case assessed. Outcomes one year later included children remaining with biological family and children remaining on a child protection plan.

Results A total of 67 safeguarding cases were seen during that period of time. $78 \%$ were seen in an OP setting. Median age $=5$ yrs 7 months (IQR 2 to 9 years). $\mathrm{OOH}$ median age $=20$ months (IQR -12 to 35 months). Median waiting times and type of abuse were similar. Twenty eight percent were on a child protection plan (CPP) at the time of referral to the OP service versus $9 \%$ for $\mathrm{OOH}$ referrals. Fractures and burns were more often seen in the $\mathrm{OOH}$ setting whereas no injury seen was more often documented in the OP setting.

Conclusions The type of safeguarding cases seen do differ between acute and community settings in the age of presentation, the type of injury seen, the presence of history taking from the child and the numbers on a child protection plan at the point of referral. However the type of abuse, whether physical, neglect or sexual is similar. Further work needs to be done on standardising safeguarding practice across acute and community settings.

\section{REFERENCES}

1 The Munro Review of Child protection. Department of Education. 2011

2 The Protection of Children in England: A Progress Report. Department of Education. 2009 